\title{
Expression of BMP2, TLR3, TLR4 and COX2 in colorectal polyps, adenoma and adenocarcinoma
}

\author{
LI XIANG $^{1}$, SHIQI WANG ${ }^{1}$, XIANQING JIN ${ }^{1}$, WENJUAN DUAN ${ }^{1}$, XIONGHUI DING $^{1}$ and CHANG ZHENG $^{2}$ \\ ${ }^{1}$ Department of Ministry of Education Key Laboratory of Child Development and Disorders and Department of \\ Neonatal Gastrointestinal Surgery, Children's Hospital of Chongqing Medical University; ${ }^{2}$ Department of General \\ Surgery, The Second Affiliated Hospital of Chongqing Medical University, Chongqing 400014, P.R. China
}

Received May 8, 2012; Accepted August 13, 2012

DOI: $10.3892 / \mathrm{mmr} .2012 .1046$

\begin{abstract}
The initiation and development of colorectal cancer is closely associated with the malignant transformation of colorectal polyps. The aim of this study was to analyze the expression of the bone morphogenetic protein-2 (BMP2), toll-like receptor 3 (TLR3), TLR4 and cyclooxygenase-2 (COX2) proteins in colorectal polyps, adenoma and adenocarcinoma. An immunohistochemical streptavidin-peroxidase (SP) method was used to examine the expression of MBP2, TLR4, TLR3 and COX2 in 20 colorectal juvenile polyps and 15 colorectal polyps of hamartomatous polyposis obtained from children, and 20 colorectal adenomas and 20 colorectal adenocarcinomas obtained from adults. A comparison of the expression levels of TLR3 among the groups revealed a gradual downward trend from the colorectal juvenile polyp group to the colorectal hamartomatous polyposis, adenoma and adenocarcinoma groups, respectively. The expression level of TLR3 was significantly lower in the colorectal adenocarcinoma group $(\mathrm{p}<0.05)$. The expression levels of TLR3, TLR4 and BMP2 were significantly different among the colorectal juvenile polyp, hamartomatous polyposis, adenoma and adenocarcinoma groups. These three protein molecules may be significant in the development and malignant transformation of colorectal polyps.
\end{abstract}

\section{Introduction}

Colorectal cancer is a disease that is detrimental to human health. The rate of colorectal cancer incidence in China has increased year by year. The mortality rate for colorectal

Correspondence to: Professor Xianqing Jin, Children's Hospital of Chongqing Medical University, Department of Neonatal Gastrointestinal Surgery, 136 Zhongshan 2nd Road, Yuzhong, Chongqing 400014, P.R. China

E-mail: etzhl@163.com

Key words: colorectal juvenile polyp, colorectal hamartomatous polyposis, colorectal adenoma, colorectal adenocarcinoma, toll-like receptor 4 cancer has risen to rank fourth among deaths caused by malignant tumors in China. Previous studies have shown that the initiation and development of colorectal cancer is closely associated with the malignant transformation of colorectal polyps, particularly in colorectal hamartomatous polyposis (hereditary hamartomatous polyposis syndrome, HHPS) (1). An investigation by Morson et al revealed that $50-70 \%$ of colorectal cancer originates from adenomas and that the rate of malignant transformation from multiple familial polyps is $100 \%$ (2). A high degree of villous composition in adenomas is associated with a high rate of malignant transformation (3). Colorectal polyps are a common gastrointestinal disease in children. According to pathological histology, colon polyps are divided into several subtypes, including colorectal juvenile polyps and colorectal hamartomatous polyposis (including juvenile polyposis, Peutz-Jeghers polyps and familial adenomatous polyposis syndrome). The malignant transformation of colorectal polyps may be induced by multiple factors, involves multiple genes and is a complex pathological process that proceeds through multiple stages. We utilized an immunohistochemical approach to examine the expression profiles of the molecular markers toll-like receptor 3 (TLR3) and TLR4 (inflammation response factors), bone morphogenetic protein 2 (BMP2; a cell differentiation and proliferation factor) and cyclooxygenase-2 (COX2; an enzymatic response factor) in colorectal juvenile polyp, hamartomatous polyposis, adenoma and adenocarcinoma tissues and identified the molecular markers that indicate the early malignant transformation of colorectal polyps.

\section{Materials and methods}

Clinical data of patients and details of the tissue specimens. Twenty children diagnosed with colorectal juvenile polyp and 15 children diagnosed with colorectal hamartomatous polyposis (including 9 juvenile polyposis cases; 2 PentzJeghers syndrome cases and 4 familial adenomatous polyposis syndrome cases) were treated at the Children's Hospital of Chongqing Medical University between June 2006 and June 2011. Twenty adult patients diagnosed pathologically with colorectal adenoma and 20 adult patients diagnosed pathologically with colorectal adenocarcinoma were selected at random from patients who were treated at The Second 
Affiliated Hospital of Chongqing Medical University between June 2006 and June 2011. All diagnoses were confirmed by pathological examination and all patients and the parents of the children provided informed consent for tumor preservation and biological analysis prior to surgery. The following patient characteristics were recorded: i) the children comprised 25 boys and 10 girls of which 6 were aged 1-3 years, 20 were aged 3-6 years and 9 were aged 6-12 years; ii) the adult patients were 27 men and 13 women, of which 2 were 20-30 years old, 2 were 30-40 years old, 11 were $40-50$ years old, 15 were 50-60 years old, 5 were 60-70 years old and 5 were $70-80$ years old. The tissue specimens were obtained from the rectum (45 cases), the sigmoid colon (11 cases), the descending colon (3 cases), the transverse colon (11 cases) and the ascending colon (5 cases). Consent was obtained either from the patient or the patient's family.

Immunohistochemistry. An immunohistochemical streptavidin-peroxidase (SP) method was used. Staining was performed by strictly following the manufacturer's instructions for the staining kit. A positive biopsy provided in the kit was used as the positive control. Phosphate buffer was used instead of the primary antibody as the negative control. The tissue sections were dewaxed and rehydrated and the sections were treated with $3 \%$ hydrogen peroxide for $5 \mathrm{~min}$ to inactivate endogenous peroxidase. The antigens were recovered by heating in a microwave and the appropriate primary antibody was then added [BMP2 (1:300), TLR3 (1:500), TLR4 (1:200) and COX2 (1:300)]. The slides were incubated at $4^{\circ} \mathrm{C}$ in a humidified chamber overnight, then incubated with a biotin-conjugated secondary antibody at $37^{\circ} \mathrm{C}$ for $30 \mathrm{~min}$. Horseradish peroxidase-linked avidin was then added and the slides were incubated at $37^{\circ} \mathrm{C}$ for $30 \mathrm{~min}$. The substrate diaminobenzidine (DAB) was added to develop the color and the slides were dehydrated and mounted. The image analysis software Image Pro Plus was used to semi-quantitatively analyze the images. Five high-magnification (x400) areas of high-quality staining were randomly selected for every sample. An integrated optical density (IOD) value was calculated for the positively stained cells. An average of 5 viewing areas was used to determine the OD value for the positive cells of the sample.

Statistical analysis. The data were analyzed with the statistical software SPSS 13.0 and are presented as the mean \pm standard deviation $( \pm \mathrm{s})$. The groups were compared using the Student's $\mathrm{t}$-test and $\mathrm{p} \leq 0.05$ was considered to indicate a statistically significant result.

\section{Results}

Expression of TLR4 in the colorectal juvenile polyp, hamartomatous polyposis, adenoma and adenocarcinoma groups. In the glandular tissue of colorectal juvenile polyps, TLR4 was weakly expressed or was not expressed at all. However, TLR4 expression was significantly enhanced in cancerous tissue and displayed a diffuse or granular distribution on the cell membrane and in the cytoplasm but was absent from the nucleus (Fig. 1A). The expression levels of TLR4 in the colorectal juvenile polyp and colorectal adenoma groups were significantly lower than those in the colorectal hamartomatous polyposis and colorectal adenocarcinoma groups. We observed no significant difference between the juvenile polyp and adenoma groups ( $p>0.05)$ and no significant difference between the hamartomatous polyposis and adenocarcinoma groups ( $p>0.05)$. However, significant differences between the juvenile polyp and hamartomatous polyposis groups $(\mathrm{p}<0.05)$ and between the adenoma and adenocarcinoma groups $(p<0.05)$ were observed (Fig. 2).

Expression of BMP2 in the colorectal juvenile polyp, hamartomatous polyposis, adenoma and adenocarcinoma groups. BMP2 was expressed in the glandular polyp tissue of colorectal juvenile polyps and adenomas as uniform diffuse brown positive staining in the cytoplasm (Fig. 1B). The expression levels of BMP2 in the colorectal juvenile polyp and adenoma groups were significantly higher than those in the colorectal hamartomatous polyposis and adenocarcinoma groups. We observed no significant differences among the colorectal juvenile polyp, hamartomatous polyposis and adenoma groups $(\mathrm{p}>0.05)$. However, the expression levels of BMP2 were significantly lower in the colorectal adenocarcinoma group ( $<<0.05$; Fig. 2).

Expression of TLR3 in the colorectal juvenile polyp, hamartomatous polyposis, adenoma and adenocarcinoma groups. The expression of TLR3 on the cell membrane and in the cytoplasm in the colorectal juvenile polyps was significantly higher than that in the other groups (Fig. 1C). The expression levels of TLR3 revealed a gradual downward trend from the colorectal juvenile polyp group to the colorectal hamartomatous polyposis, adenoma and adenocarcinoma groups, respectively. We observed no significant differences among the colorectal juvenile polyp, hamartomatous polyposis and adenoma groups $(\mathrm{p}>0.05)$. However, the expression level of TLR3 was significantly lower in the colorectal adenocarcinoma group (p<0.05; Fig. 2).

Expression of COX2 in the colorectal juvenile polyp, hamartomatous polyposis, adenoma and adenocarcinoma groups. No significant differences in COX 2 expression levels were observed among the 4 treatment groups ( $p>0.05$; Fig. 1D and Fig. 2).

\section{Discussion}

TLRs are important membrane receptors that not only are involved in tissue development and maintenance but also have critical functions in the innate immune response. TLRs are able to recognize the highly conserved molecular structure known as the pathogen-associated molecular pattern (PAMP), which commonly exists in one or multiple specific types of microbial pathogens and their metabolic products. The recognition of PAMPs by TLRs initiates an early response to target the invading pathogen and subsequently induce the acquired immune response. The ligands of TLRs are primarily specific metabolic products from microorganisms. TLR3 predominantly recognizes dsDNA from viruses and parasites while TLR4 particularly recognizes LPS of Gram-negative bacteria (4). The same PAMP may trigger different TLRs to produce different cytokines, which suggests that invasion by 

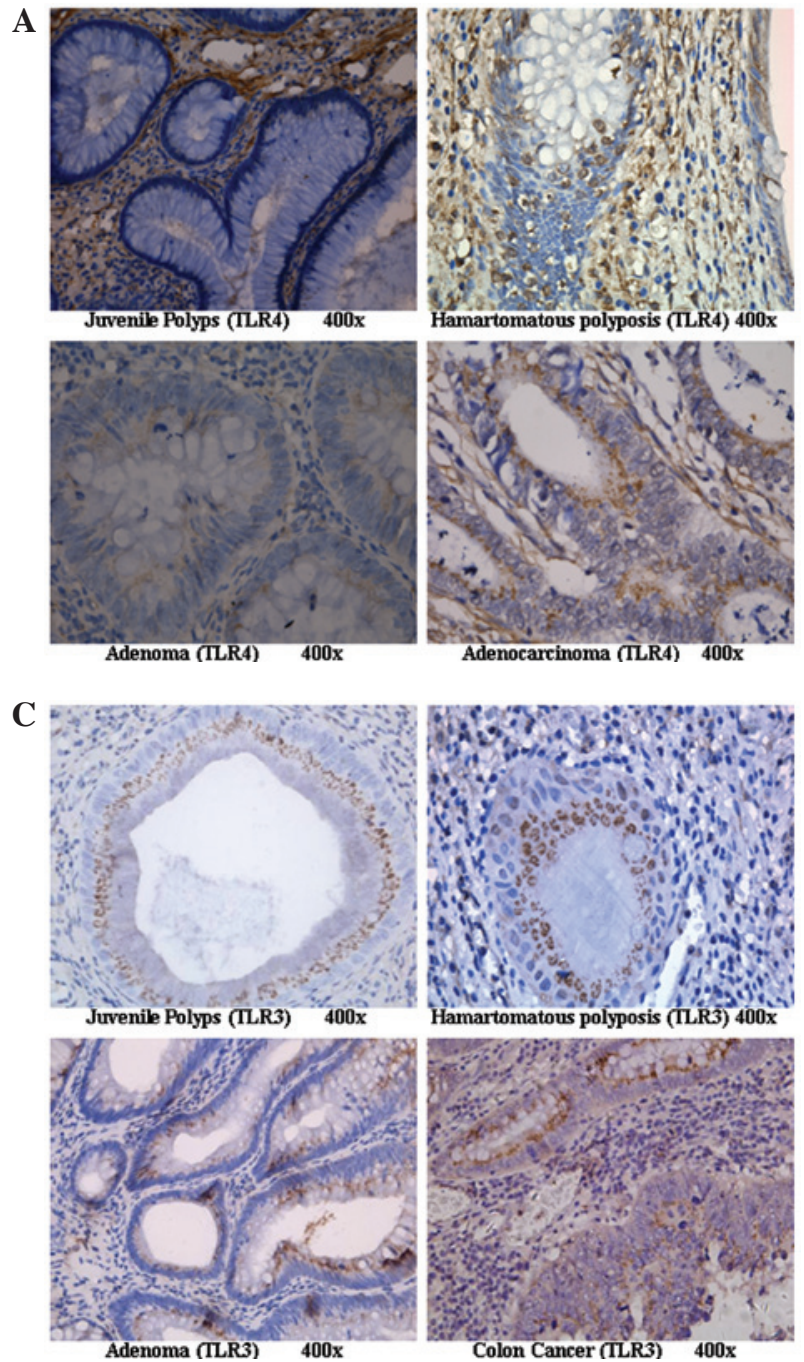

B
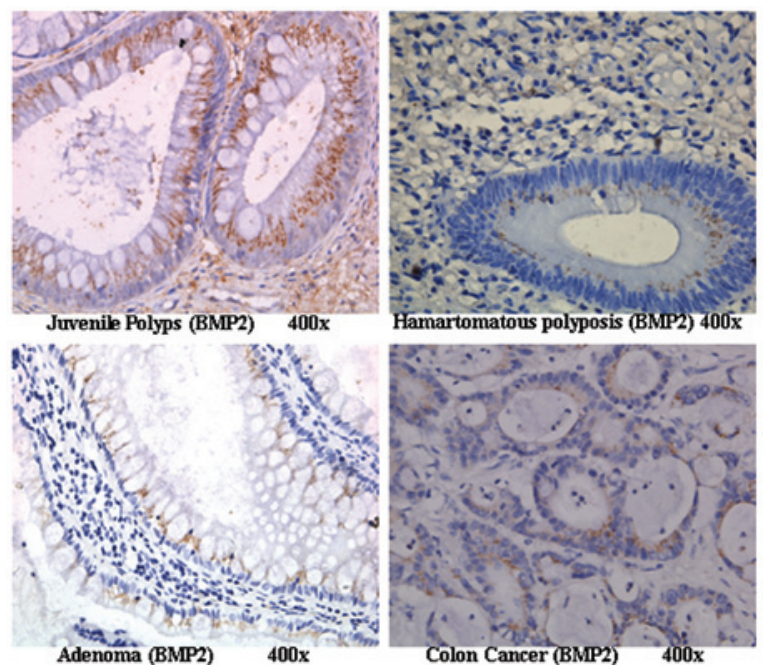

D
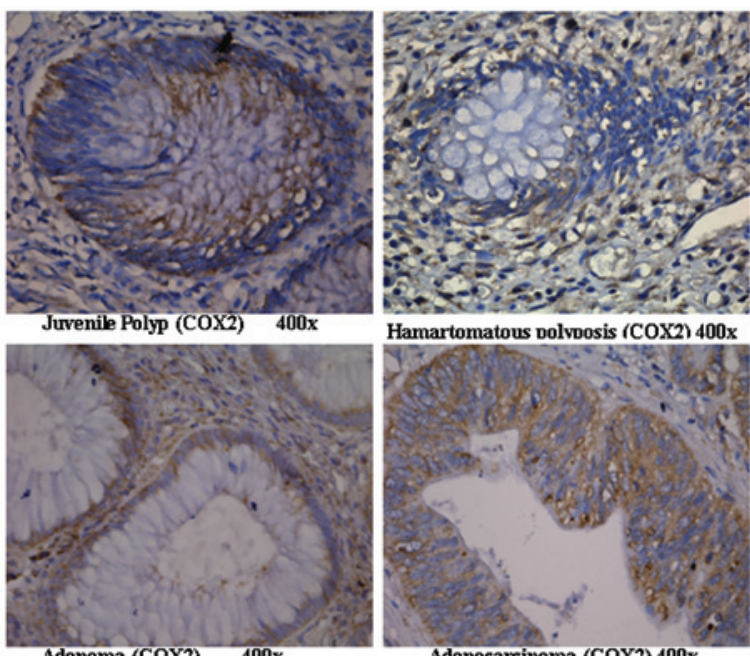

Adenocareinoma (COX2) 400x

Figure 1. Expression of toll-like receptor (TLR) 3 and 4, bone morphogenetic protein-2 (BMP2) and cyclooxygenase-2 (COX2) in the different groups. (A) TLR4 was weakly expressed in the glandular tissue of colorectal juvenile polyps; enhanced expression of TLR4 was observed in carcinoma tissue in a diffuse or granular distribution on the cell membrane and in the cytoplasm. (B) BMP2 was expressed in the glandular polyp tissue of colorectal juvenile polyps and adenomas as uniform diffuse brown positive staining in the cytoplasm, while it was weakly expressed in colorectal hamartomatous polyposis and adenocarcinoma tissue. (C) The expression of TLR3 on the cell membrane and in the cytoplasm was significantly higher in the juvenile polyps than in the other groups and the expression of TLR3 was significantly decreased in colorectal adenocarcinoma. (D) The expression of COX2 did not significantly differ among groups.

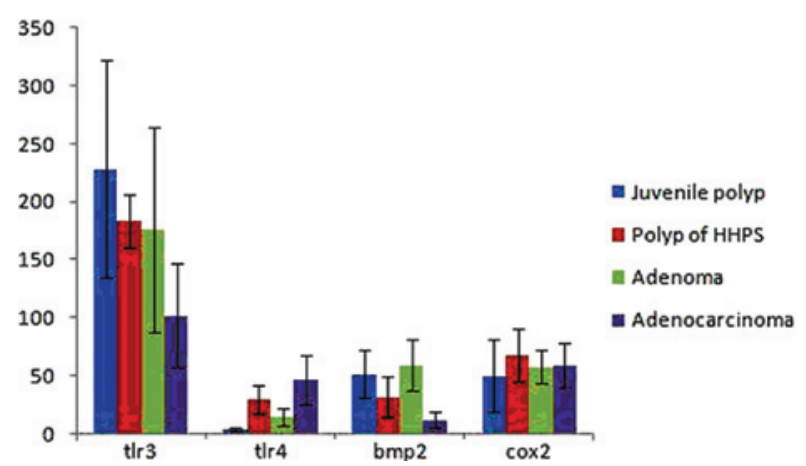

Figure 2. Expression of toll-like receptor 4 (TLR4) in the colorectal juvenile polyp and adenoma groups was significant lower than that in the colorectal hamartomatous polyposis and adenocarcinoma groups. However, the expression of bone morphogenetic protein-2 (BMP2) in the colorectal juvenile polyp and adenoma groups was significantly higher than that in the colorectal hamartomatous polyposis and adenocarcinoma groups. The expression levels of TLR3 revealed a gradual downward trend from the colorectal juvenile polyp group to the colorectal hamartomatous polyposis, adenoma and adenocarcinoma groups, respectively. HHPS, hereditary hamartomatous polyposis syndrome. the same pathogen may stimulate multiple TLRs and diversify the TLR-induced innate immune response. The immune system uses this mechanism to powerfully and effectively remove pathogens. Chronic infection and inflammation may lead to tumorigenesis and certain pathogens induce tumorigenesis through TLRs (5). Previous studies have shown that TLR3 stimulated by its ligand participates in tumor cell migration. Polyinosinic:polycytidylic acid (poly I:C) is recognized by TLR 3 and induces the maturation of dendritic cells (DCs), which effectively stimulates the immune response (6). Upregulation of the expression of TLR4-induced signal transduction factors in intestinal carcinoma cells may stimulate the production of immunosuppressive factors, including TGF- $\beta$ and VEGF, and modify the local microenvironment of the tumor to facilitate carcinoma cell escape, proliferation, invasion and metastasis (7). Our investigation revealed that in the glandular tissue of juvenile polyps, TLR4 was weakly expressed or was not expressed at all. However, TLR4 expression was significantly enhanced in cancer tissue and displayed 
a diffuse or granular distribution on the cell membrane and in the cytoplasm but was absent from the nucleus. The increased expression of TLR4 in the colorectal hamartomatous polyposis and adenocarcinoma groups was significant $(\mathrm{p}<0.05)$. The expression of TLR3 on the cell membrane and in the cytoplasm in the juvenile polyp group was significantly higher than that in the colorectal hamartomatous polyposis, adenoma and adenocarcinoma groups $(\mathrm{p}<0.05)$. We suggest that TLR3 and TLR4 are important markers to indicate the early malignant transformation of colorectal polyps. The difference between the two TLRs suggests that TLR-mediated mechanisms, in addition to the inflammation-induced response, may contribute to tumor initiation. Bendelac et al proposed a "danger model" theory for tumor tissue (8). The ligands for TLRs on the cell surface of antigen-presenting cells represent a subset of the danger signals. Following the initiation of a specific antitumor immune response, tumor cells are constantly killed by cytotoxicity, which subsequently causes the continuous production of the danger signals. Therefore, TLRs are able to continuously stimulate the antibody-tumor immune response. However, some researchers consider that toll receptors participate in the mechanism by which tumor cells escape immune surveillance (9). Tumor cells use TLRs expressed on the cell surface to generate a tumor-promoting microenvironment and escape the immune attack.

BMPs were originally discovered as proteins that induce bone and cartilage formation. However, subsequent studies have shown that BMPs are widely distributed in multiple tissues and cell types and have multiple functions, including participation in embryo formation and development and cell differentiation and proliferation. BMP signal transduction may be significant in intestinal cell differentiation (10). Hardwick et al found that BMP2 inhibits colon epithelial cell growth in vitro, induces apoptosis and inhibits cell proliferation (11). BMP2 expression was detected in the epithelial cells of normal adults and of mice; however, BMP2 expression was lost in the micro adenomas of patients with familial adenomatous polyps. In the current study, BMP2 expression in the glandular tissue of the polyps was observed as uniformly diffuse brown positive staining in the cytoplasm and was weakly stained positive in cancer tissue. The trend towards the significant decrease in the expression of BMP2 from the colorectal juvenile polyp group to the colorectal hamartomatous polyposis, adenoma and adenocarcinoma groups, respectively, suggests that the loss of BMP2 causes hyperplasia of intestinal epithelial cells and tumorigenesis.

COX2 is a 604-amino acid, rate-limiting enzyme that converts arachidonic acid into prostaglandins (PGs). Under normal conditions, COX2 is not expressed in the majority of tissues but is stimulated when cells are triggered by endogenous and exogenous signals. Therefore, COX2 is recognized as a "rapid response gene" (12). COX2 gene overexpression may enhance the adhesion of intestinal epithelial cells to the extracellular matrix, extend the G1 phase of the cell cycle 3 -fold, inhibit apoptosis, trigger a series of gene mutations, and eventually lead to tumor growth. Previous studies have demonstrated that $\mathrm{COX} 2$ expression is closely correlated with the malignant transformation of intestinal polyps and that COX 2 may be used as a molecular marker for the early malignant transformation of intestinal polyps (13). Our study shows that COX2 expression does not significantly differ among the various groups. We propose that $\mathrm{COX} 2$ expression does not change significantly during the transition from polyps to adenoma and adenocarcinoma.

In summary, our current study demonstrates that there are significant differences among the expression levels of TLR3, TLR4 and BMP2 in colorectal juvenile polyps, hamartomatous polyposis, adenomas and adenocarcinomas. These three proteins may be significant in the development and the malignant transformation of colorectal polyps.

\section{References}

1. Half E, Gldberg Y, Kariv R, et al: Guidelines for diagnosis, treatment, surveillance and prevention of cancer in patients with familiar non-adenomatous polyposis. Harefuah 150: 60, 2011.

2. Morson BG: Genesis of colorectal cancer. Clin Gastroenterol 5: 505-507, 1976

3. Winawer SJ, Zauber AG, Fletcher RH, et al: Guidelines for colonoscopy surveillance after polypectomy. A consensus update by the US Multi-Society Task Force on Colorectal Cancer and the American Cancer Society. Gastroenterol 130: 1872-1885, 2006.

4. Takeda K, Kaisho T and Akira S: Toll-like receptors. Annu Rev Immunol 21: 335-367, 2003.

5. He X and Bai H: Current progress on the investigation of Toll-like receptor. Northwest National Defense Medical Journal 31: 445-446, 2010 (In Chinese).

6. Salaun B, Coste I, Rissoan MC, et al: TLR3 can directly trigger apoptosis in human cancer cells. J Immunol 176: 4894-4901, 2006.

7. Jin H and Meng Q: Measurement of TLR4 expression in human colon cancer by semi quantitative RT-PCR. Journal of Modern Applied Medicine 21: 682-684, 2009 (In Chinese).

8. Bendelac A and Medzhitov R: Adjuvants of immunity: harnessing innate immunity to promote adaptive immunity. J Exp Med 195: F19-F23, 2002

9. He L, Zhang L, Li Z and Zhang Q: The roles of toll-like receptors in carcinogenesis and cancer immunotherapy. Chin Ger Journal Clin Oncol 9: 118-120, 2010.

10. Fiocchi C: TGF-beta/Smad signaling defects in inflammatory bowel disease: mechanisms and possible novel therapies for chronic inflammation. J Clin Invest 108: 523-526, 2001.

11. Hardwick JC, Van Den Brink GR, Bleuming SA, et al: Bone morphogenetic protein 2 is expressed by, and acts upon, mature epithelial cells in the colon. Gastroenterology 126: 111-121, 2004.

12. Zhang F, Warskulat U, Wettstein M, et al: Hyperosmolarity stimulates prostaglandin synthesis and cyclooxygenase-2 expression in activated rat liver macrophages. Biochem J 312: 135-143, 1995.

13. Wasilewicz MP, Kołodziej B, Bojułko T, et al: Expression of cyclooxygenase-2 in colonic polyps. Pol Arch Med Wewn 120: 313-320, 2010. 